\title{
On the Spectral Efficiency of CDMA Downlink Cellular Mobile Networks
}

\author{
Khairi Ashour Hamdi, Senior Member, IEEE, and Pongsatorn Sedtheetorn, Member, IEEE
}

\begin{abstract}
A new accurate mathematical analysis is presented for the efficient computation of the spectral efficiency of CDMA downlink wireless communication systems in the presence of multipath Rayleigh fading and log-normal shadowing. A new explicit expression is derived for the spectral efficiency, which is based on an accurate interference model that accounts for both intra-cell and inter-cell interferences. This is used to investigate the performance of downlink CDMA in different multipath delay profiles. The numerical results has shown that the loss of orthogonality deteriorates the spectral efficiency significantly at high signal-to-noise ratios (SNR) levels. On the other hand, the diversity gain will compensate the loss of orthogonality at low SNR levels.
\end{abstract}

Index Terms-Spectral efficiency, CDMA, orthogonality factor, self interference, Rayleigh fading.

\section{INTRODUCTION}

$\mathbf{T}$ HIS paper presents a new accurate analysis for the spectral efficiency of downlink CDMA multi-cellular mobile communication systems. Previous research results on CDMA cellular mobile networks have not provided accurate, computationally efficient explicit expressions for computing the spectral efficiency in CDMA downlink multi-cellular systems.

The difficulty of the present problem can be explained as follows. The signal-to-interference-plus-noise-ratio (SINR) experienced by an arbitrary mobile in a downlink CDMA system is a ratio of a mixture of non-identical random variables. Moreover, the presence of multipath fading leads to the lost of orthogonality between signals transmitted by the same base station, which results in additional self interference. The existence of this self-interference renders the problem of computing the spectral efficiency even more difficult, as the SINR becomes now a ratio of non-independent random variables. Direct methods to compute the average spectral efficiency in this case would require at least $K$-fold integrations ( $K$ is the number of neighboring cells). Consequently, it is quite common in the previous research on the spectral efficiency of downlink CDMA to approximate the intercell interference by its average (e.g. [1]-[4]). Other common methods include considering only an isolated cell environment [5], Monte Carlo simulation [6], or bounding techniques [7].

Manuscript received September 5, 2007; revised January 28, 2008; accepted March 26, 2008. The associate editor coordinating the review of this paper and approving it for publication was I.-M. Kim.

K A Hamdi is with the School of Electrical \& Electronic Engineering, The University of Manchester, Sackville Street, Po Box 88, Manchester, M60 1QD, United Kingdom (e-mail: k.hamdi@manchester.ac.uk).

P. Sedtheetorn was with the School of Electrical and Electronic Engineering, University of Manchester, U.K. He is now with the Department of Electrical and Computer Engineering, Mahidol University, Phuttamonthon Nakomprathom 73170, Thailand (e-mail: egpst@mahidol.ac.th).

Digital Object Identifier 10.1109/T-WC.2008.070990

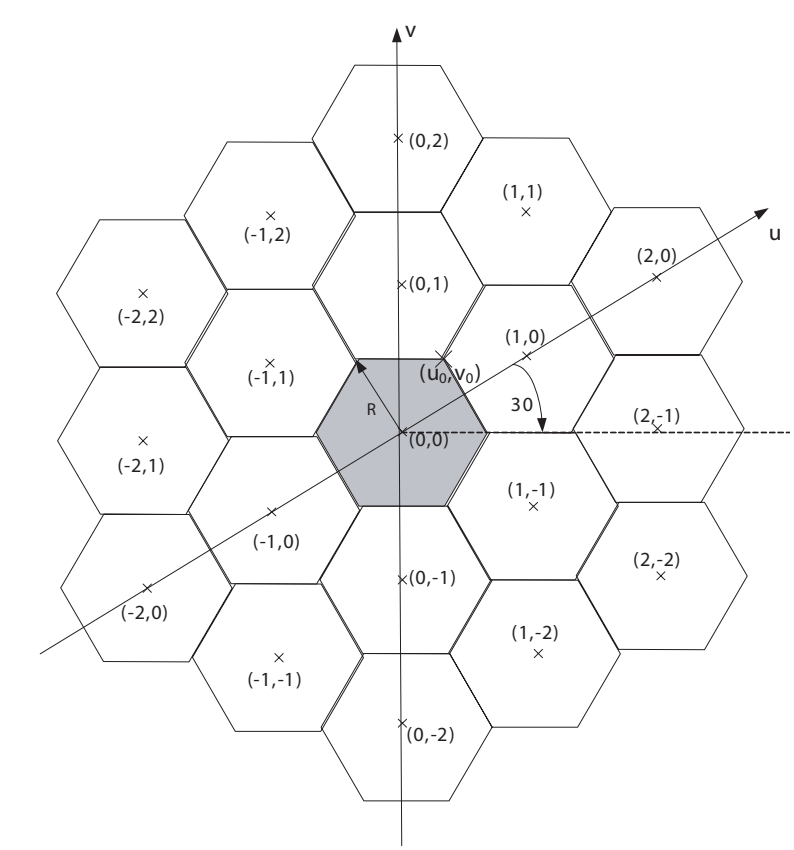

Fig. 1. oordinates of the co-channel cells in the first and second tier on $u v$-plane. The interfered mobile is located at $\left(u_{0}, v_{0}\right)$ with its home base station being at the origin. Note that the scale of both $u$ and $v$ axes is 1 unit $=\sqrt{3} R$.

In this paper, based on an accurate SINR model that accounts for both intercell and intra-cell interference, we derive a new computationally efficient explicit expression for the spectral efficiency of a CDMA downlink in a multipath Rayleigh fading environment. This reduces significantly the required computational complexity required to compute the spectral efficiency by direct methods, and thus facilitates accurate evaluation of the impacts of the lost of orthogonality on the overall efficiency of a downlink CDMA. The main contribution of this paper is therefore to provide a simple explicit expression for the spectral efficiency of downlink CDMA. To the best of our knowledge, no such simple analytical expressions for the spectral efficiency are available in the open literature.

The rest of this paper is organized as follows. Section II describes the CDMA downlink interference model. The spectral efficiency analysis is presented in Section III. The extension of the basic results to include log-normal shadow fading is include in Section IV. Simulation and numerical results are given in Section V, and Section VI concludes the paper.

\section{DOWNLINK SINR ANALYSIS}

Consider a downlink CDMA multicellular system in a frequency-selective Rayleigh fading channel, Fig. 1, and let the composite CDMA downlink signal comprises $M$ mutually 


$$
\mathrm{SINR}_{m}=\frac{P_{n} d_{0, m}^{-\beta} \sum_{l=1}^{L}\left|h_{l, 0}\right|^{2}}{\Gamma \frac{P-P_{m}}{G} d_{0, m}^{-\beta} \sum_{l=1}^{L}\left|h_{l, 0}\right|^{2}+\frac{P}{G} \sum_{k=1}^{K} \sum_{l=1}^{L} d_{k, m}^{-\beta}\left|h_{l, k}\right|^{2}+N_{0}}, m=1,2, \ldots, M
$$

orthogonal signals, which are destined to $M$ different mobiles in the same cell. Though these signals appear orthogonal at the transmitter side, however, the orthogonality is lost at the receiver side after being propagated over a multipath fading environment, resulting in intra-cell interference (self interference). This interference adds to the white Gaussian noise (AWGN) and the intercell interference from neighboring cells. Therefore, the signal-to-interference-plus-noise-ratio (SINR) experienced by an arbitrary mobile, say mobile $m$, at the output of the Rake receiver is a random variable, that can be written as shown in (1). (e.g. [13])

Here, $K$ is the number of neighboring cells, $G$ is the processing gain (a constant parameter, which equals to the number of chips per bit), and $L$ is the number of faded paths. It is worth mentioning that the number of paths $L$ is related to the maximum delay spread of the channel and is assumed to be less than the processing gain $G . h_{l, k}, l=1, . . L$, $k=0,1,2, . ., K$ are mutually independent zero-mean complex Gaussian path gains, with $\mathbb{E}\left[\left|h_{l, k}\right|^{2}\right]=\alpha_{l}$, that models the multipath Rayleigh fading channel.

$P_{m}$ in (1) is the transmit power allocated to the reference mobile, and $P$ is the total transmit power in the cell. $N_{0}$ is the variance of the AWGN, and $\beta$ is the path loss exponent.

$d_{k, m}, k=0,1, \ldots, K$, in (1) represent the distance between mobile $m$ (in cell 0 ) and the $k$ th base station $(k=0$ for the home base station). Thus, the term $d_{k, m}^{-\beta}, k=0,1, . ., K$, in (1) represents the mean path gain between the $k$ th base station and mobile $m$. In order to find an expressions for $d_{k, m}$, consider the geometry of hexagonal cells depicted in Fig. 1, and let $\left(x_{m}, y_{m}\right)$, be the coordinates (in the hexagonal coordinates system) of mobile $m$ in cell 0 , and $\left(u_{k}, v_{k}\right), k=1,2, \ldots, K$, be the coordinates of the $k$ th neighboring base station. Then, it can be shown that $d_{0, m}=\sqrt{3} R \sqrt{x_{0}^{2}+y_{0}^{2}+x_{0} y_{0}}$, with $R$ being the cell radius, whereas $d_{k, m}$ for $k>0$ is given in (2) [9].

The coefficient $\Gamma$ appearing at the denominator of (1) is the so called orthogonality factor (OF), which represents the instantaneous fraction of received downlink power that gets converted by multipath into intra-cell interference. It is a random variable that depends on the paths' gains, and given by

$$
\Gamma=1-\frac{\sum_{l=1}^{L}\left|h_{l, 0}\right|^{4}}{\left(\sum_{l=1}^{L}\left|h_{l, 0}\right|^{2}\right)^{2}}
$$

Recent research results (e.g. [10]-[13]) have suggested that $\Gamma$ can be accurately approximated by an (approximate) of its average $^{1}$

$$
\Gamma \approx 1-\frac{\sum_{l=1}^{L} \alpha_{l}^{2}}{\left(\sum_{l=1}^{L} \alpha_{l}\right)^{2}}
$$

However, even when $\Gamma$ in (3) is replaced by its average (4), the problem of computing averages involving SINR is still not trivial. In order to explain in more details the difficulty associated with computing averages involving SINR, notice that SINR in (1) involves a ratio of $(K+1) L$ random variables. Moreover, the numerator and denominator are not independent (because of the appearance of the random variables $\sum_{l=1}^{L}\left|h_{l, 0}\right|^{2}$ at both the numerator and the denominator). It is also important to emphasize, at this point, that the term $\sum_{k=1}^{K} \sum_{l=1}^{L} d_{k, m}^{-\beta}\left|h_{l, k}\right|^{2}$ which appears at the denominator of (1), and represents the accumulated interference from the neighboring cells, is in fact a sum of exponential random variables with arbitrarily non-equal means, for which exact analytical expressions of its probability density function (pdf) are not known in closed-form (e.g. [15], [16]). This would complicate very much the task of deriving explicit expressions for the pdf of SINR. On the other hand, the direct convolution approach may require at least $K$-fold convolutional integrals. Because of this difficulty, it is quite common in some other research to approximate the intercell interference term $\sum_{k=1}^{K} \sum_{l=1}^{L} d_{k, m}^{-\beta}\left|h_{l, k}\right|^{2}$ by its mean (e.g. $I_{\mathrm{oc}}$ in [13, Eq. 1]).

In next section, we present a non-direct approach which facilitates exact computation of the average of the function $\ln (1+$ SINR $)$ without needing an explicit expression the SINR's pdf.

\section{THE SPECTRAL EFFICIENCY}

The spectral efficiency is a measure of the average number of information bits that can be transmitted arbitrarily reliably per second per Hertz per cell. Let $M$ be the number of mobiles per cell. Then, the spectral efficiency per cell, is given by [6], [14]

$$
C=\frac{1}{G} \sum_{m=1}^{M} C_{m}
$$

where $C_{m}$ is given in (6) and $\mathbb{E}$ is the expectation operator. $\mathrm{SNR}_{m}=\frac{P_{m} d_{m, 0}^{-\beta}}{N_{0}}$ is the average signal-to-noise (SNR) experienced by mobile $m$ in the absence of interference, and $\rho_{m}=\frac{P_{m}}{P}$ is the fraction of power allocated to mobile $m$, which depends on the employed power allocation strategy. In the special case of uniform power allocations. $\rho_{m}=\frac{1}{M}, m=$ $1,2, \ldots M$.

\footnotetext{
${ }^{1}$ It is worth noticing that (4) is not the true average of (3).
} 


$$
\begin{gathered}
d_{k, m}=\sqrt{3} R \sqrt{\left(u_{k}-x_{m}\right)^{2}+\left(v_{k}-y_{m}\right)^{2}+\left(u_{k}-x_{m}\right)\left(v_{k}-y_{m}\right)}, k=1,2, \ldots, K \\
C_{m}=\mathbb{E}\left[\log _{2}\left(1+\frac{\rho_{m} \sum_{l=1}^{L}\left|h_{l, 0}\right|^{2}}{\frac{1-\rho_{m}}{G} \Gamma \sum_{l=1}^{L}\left|h_{l, 0}\right|^{2}+\frac{1}{G} \sum_{k=1}^{K} \sum_{l=1}^{L}\left[\frac{d_{m, k}}{d_{m, 0}}\right]^{-\beta}\left|h_{l, k}\right|^{2}+\frac{\rho_{m}}{\mathrm{SNR}_{m}}}\right)\right]
\end{gathered}
$$

The average in (6) is with respect to set of exponential random variables $\left|h_{l, k}\right|^{2}, l=1,2, \ldots, L, k=0,1, \ldots, K$. Direct computations of the average in (6) requires an explicit expression for the probability density functions of the random variables SINR, defined in (1) which, as explained at the end of the last section, is difficult to obtain in closed form when the number of neighboring cells $K>1$. In this paper, we present an original non-direct method for efficient computation of the average in (6). This is based on the following lemma.

Lemma 1: Let $u$ be a random variable having a pdf $f(u ; a, m)=\frac{u^{m-1}}{\Gamma(m)} a^{-m} e^{-u / a}$, and let $v$ be an arbitrary nonnegative random variable that is "independent" of $u$. Then for any constants $c, d>0$, we have (7), where $\mathcal{M}_{v}(z)=\mathbb{E}\left[e^{-z v}\right]$ is the moment generating function of the random variable $v$.

Proof: The proof is given in the Appendix.

Now, as far computing the average spectral efficiency (6) is concerned, notice that the term at numerator $\sum_{l=1}^{L}\left|h_{l, 0}\right|^{2}$ has not always a gamma distribution (it would have a gamma distribution only in the special case of uniform delay profiles, where $\alpha_{1}=\alpha_{2}=\ldots=\alpha_{L}$ ), and hence Lemma 1 can not be applied straightforwardly. Therefore, in order to apply Lemma 1 for arbitrary delay profiles, we show next that it is always possible to express the pdf of $\sum_{l=1}^{L}\left|h_{l, 0}\right|^{2}$ as a weighted sum of gamma density functions. To prove this, let $x=\sum_{l=1}^{L}\left|h_{l, 0}\right|^{2}$. Then, since $\left\{\left|h_{l, 0}\right|^{2}, l=1,2, \ldots, L\right\}$ are independent exponential random variables, we have for its MGF

$$
\mathcal{M}_{x}(z)=\mathbb{E}\left[e^{-z x}\right]=\prod_{l=1}^{L} \frac{1}{1+\alpha_{i} z}=\sum_{l=1}^{L} \frac{b_{l}}{\left(1+\alpha_{l} z\right)^{m_{l}}}
$$

where we have resolved it into partial fractions. Here, $m_{l} \in\{0,1, \ldots, L\}$ accounts for the repeated roots, and $\left\{b_{1}, b_{2}, \ldots, b_{L}\right\}$ are some constants. It is worth mentioning that computing these coefficients is tedious and requires to solve a set of linear equations and computing high-order partial derivatives. Fortunately, our final expression for the average spectral efficiency does not requires computing these coefficients.

Eq. (8) implies that the pdf of $x=\sum_{l=1}^{L}\left|h_{l, 0}\right|^{2}$ can be expressed as the weighted sum

$$
f(x)=\sum_{l=1}^{L} b_{l} f\left(x ; \alpha_{l}, m_{l}\right)
$$

where $f(x ; a, m)=\frac{x^{m-1}}{\Gamma(m)} a^{-m} e^{-x / a}$ is the gamma probability density function with parameter $m$, and mean $a m$.
Now, consider the conditional average shown in (10). which, upon invoking (7), reduces into (11), where we have used (8).

We arrive at the explicit expression shown in (12) for the average spectral efficiency, where we have used

$$
\begin{aligned}
\mathcal{M}_{v}(z) & =\mathbb{E}\left[e^{-z v}\right]=\mathbb{E}\left[e^{-z \frac{1}{G} \sum_{k=1}^{K} \sum_{l=1}^{L}\left[\frac{d_{k, m}}{d_{0, m}}\right]^{-\beta}\left|h_{l, k}\right|^{2}+\frac{\rho_{m}}{\mathrm{SNR} m}}\right] \\
& =\prod_{k=1}^{K} \prod_{l=1}^{L} \frac{1}{1+\frac{1}{G}\left[\frac{d_{k, m}}{d_{0, m}}\right]^{-\beta} \alpha_{l} z} e^{-z \frac{\rho m}{\mathrm{SNR} m}}
\end{aligned}
$$

In the special case of uniform delay profile, $\alpha_{1}=\alpha_{2}=$ $\ldots=\alpha_{L}=\frac{1}{L}$, and (12) reduces into the simpler expression shown in (14).

As far the numerical evaluation of the integrals in (12) is concerned, observe that $\forall z \geq 0$,

$$
0 \leq \frac{1}{1+\left(\rho_{m}+\Gamma \frac{1-\rho_{m}}{G}\right) \alpha_{l} z} \leq \frac{1}{1+\Gamma \frac{1-\rho_{m}}{G} \alpha_{l} z} \leq 1
$$

(notice that all $\rho_{m}, G, \Gamma$, and $\alpha_{l}$ are non-negative quantities). Accordingly,

$$
0 \leq \prod_{l=1}^{L} \frac{1}{1+\Gamma \frac{1-\rho_{m}}{G} \alpha_{l} z}-\prod_{l=1}^{L} \frac{1}{1+\left(\rho_{m}+\Gamma \frac{1-\rho_{m}}{G}\right) \alpha_{l} z} \leq 1 .
$$

Furthermore, using l'Hôpital's rule, we obtain (16).

Eqs. (15) and (16) prove that the first term in (12) is bounded over the whole range of integration

$$
\begin{aligned}
0 & \leq \frac{\prod_{l=1}^{L} \frac{1}{1+\Gamma \frac{1-\rho_{m}}{G} \alpha_{l} z}-\prod_{l=1}^{L} \frac{1}{1+\left(\rho_{m}+\Gamma \frac{1-\rho_{m}}{G}\right) \alpha_{l} z}}{z} \\
& \leq \sum_{l=1}^{L} \alpha_{l}, \quad \forall z \geq 0 .
\end{aligned}
$$

It can be verified that, the second term in (12) is also bounded

$$
0 \leq \prod_{k=1}^{K} \prod_{l=1}^{L} \frac{1}{1+\frac{M}{G}\left[\frac{d_{k, m}}{d_{0, m}}\right]^{-\beta} \alpha_{l} z} e^{-z \frac{\rho_{m}}{\mathrm{SNR}_{m}}} \leq 1, \quad \forall z \geq 0 .
$$

(17) and (18) confirm that the integrand in (12) is a non-negative bounded function in the range of integration. Furthermore, it can be seen that it is also continuous and possess all derivatives $\forall z \geq 0$. Therefore, standard numerical integration packages can be used to compute (12) or (14) without any difficulty.

To summarize, (12) is a new exact explicit expression for the average in (6) represents the spectral efficiency experienced by mobile $m$ that experience a SINR given by (1), and 


$$
\begin{aligned}
& \mathbb{E}\left[\ln \left(1+\frac{d u}{c u+v}\right)\right]=\int_{0}^{\infty} \frac{1}{z}\left[\left(\frac{1}{1+a c z}\right)^{m}-\left(\frac{1}{1+a(d+c) z}\right)^{m}\right] \mathcal{M}_{v}(z) d z \\
& \mathbb{E}\left[\ln \left(1+\frac{d \sum_{l=1}^{L}\left|h_{l, 0}\right|^{2}}{c \sum_{l=1}^{L}\left|h_{l, 0}\right|^{2}+v}\right) \mid v\right]=\int_{0}^{\infty} \ln \left(1+\frac{d x}{c x+v}\right)\left\{\sum_{l=1}^{L} b_{l} f\left(x ; \alpha_{l}, m_{l}\right)\right\} d x \\
& =\sum_{l=1}^{L} b_{l} \int_{0}^{\infty} \ln \left(1+\frac{d x}{c x+v}\right) f\left(x ; \alpha_{l}, m_{l}\right) d x \\
& \mathbb{E}\left[\ln \left(1+\frac{d \sum_{l=1}^{L}\left|h_{l, 0}\right|^{2}}{c \sum_{l=1}^{L}\left|h_{l, 0}\right|^{2}+v}\right)\right]=\int_{0}^{\infty} \frac{1}{z} \sum_{l=1}^{L} b_{l}\left(\left(\frac{1}{1+\alpha_{l} c z}\right)^{m_{l}}-\left(\frac{1}{1+\alpha_{l}(d+c) z}\right)^{m_{l}}\right) \mathcal{M}_{v}(z) d z \\
& =\int_{0}^{\infty} \frac{1}{z}\left(\prod_{l=1}^{L} \frac{1}{1+\alpha_{l} c z}-\prod_{l=1}^{L} \frac{1}{1+\alpha_{l}(d+c) z}\right) \mathcal{M}_{v}(z) d z \\
& C_{m}=\left(\log _{2} e\right) \int_{0}^{\infty} \frac{1}{z}\left[\prod_{l=1}^{L} \frac{1}{1+\Gamma \frac{1-\rho_{m}}{G} \alpha_{l} z}-\prod_{l=1}^{L} \frac{1}{1+\left(\rho_{m}+\Gamma \frac{1-\rho_{m}}{G}\right) \alpha_{l} z}\right] \\
& \times \prod_{k=1}^{K} \prod_{l=1}^{L} \frac{1}{1+\frac{1}{G}\left[\frac{d_{k, m}}{d_{0, m}}\right]^{-\beta} \alpha_{l} z} e^{-z \frac{\rho_{m}}{\mathrm{SNR}_{m}}} d z
\end{aligned}
$$

accounts for both intra-cell interference resulting from the loss of orthogonality and the intercell interference from $K$ neighboring cells. Therefore (5) with (12) can be used to compute the spectral efficiency for downlink CDMA systems having arbitrary power delay profiles $\left\{\alpha_{1}, \alpha_{2}, \ldots, \alpha_{L}\right\}$ and power allocations $\left\{\rho_{1}, \rho_{2}, \ldots, \rho_{M}\right\}$.

\section{The EFFECT OF Log-Normal Shadow FADING}

Shadow fading is an important factor affecting the performance of wireless communication systems. In this Section, we extend the results of the previous section to incorporate shadow fading. Shadowing is commonly modeled by a lognormal distribution [20, Equ. 2.53]

$$
f(y)=\frac{10}{\ln (10)} \frac{1}{\sqrt{2 \pi} \sigma y} \exp \left[-\frac{\left(10 \log _{10} y-\mu\right)^{2}}{2 \sigma^{2}}\right]
$$

where $\mu(\mathrm{dB})$ and $\sigma(\mathrm{dB})$ are the mean and standard deviation of $10 \log _{10} y$, respectively. Let $\xi_{0}, \xi_{1}, \ldots, \xi_{K}$ be mutually independent $K+1$ lognormal random variables that models shadow fading of the relevant transmission paths. Then (6) becomes (20).

We can condition on the random variables $\left\{\xi_{0}, \xi_{1}, \ldots, \xi_{K}\right\}$ and obtain from (12) the expression shown in (21) for the conditional capacity

Therefore, the average spectral efficiency is obtained by averaging (21) with respect to the $K+1$ independent longnormal random variables $\left\{\xi_{0}, \xi_{1}, \ldots, \xi_{K}\right\}$. To this end, we in- voke a common approximation technique which is commonly used to evaluate averages of arbitrary function $g($.$) involving$ log-normal random variables in terms of Gauss-Hermite series [20]

$$
\begin{aligned}
& \mathbb{E}[g(\xi)] \\
& =\int_{-\infty}^{\infty} g\left(\xi_{k}\right) \frac{10}{\ln (10)} \frac{1}{\sqrt{2 \pi} \sigma \xi} \exp \left[-\frac{\left(10 \log _{10} \xi-\mu\right)^{2}}{2 \sigma^{2}}\right] d \xi \\
& =\int_{-\infty}^{\infty} g\left(10^{(\sqrt{2} \sigma y+\mu) / 10}\right) \frac{1}{\sqrt{\pi}} \exp \left[-y^{2}\right] d y \\
& \approx \sum_{n=1}^{N} \frac{w_{n}}{\sqrt{\pi}} g\left(10^{\left(\sqrt{2} \sigma a_{n}+\mu\right) / 10}\right)+R_{N}
\end{aligned}
$$

where $w_{n}$ and $a_{n}$ are the weights and abscissas of the $N$ th order Hermite polynomial and are tabulated in [18, Tbl. 25.10]. $R_{N}$ is the remainder term that decreases as $N$ increases.

We obtain from (22) and (21), the expression shown in (23) for the average spectral efficiency in the presence of lognormal shadow fading.

\section{NumERICAL EXAMPLES}

In this section, we present some numerical examples to demonstrate the application of the newly derived results on the performance of downlink CDMA in multipath Rayleigh fading environments. Let the home cell be surrounded by $K=18$ neighboring cells comprising the first and second tiers in a 


$$
\begin{aligned}
& C_{m}=\left(\log _{2} e\right) \int_{0}^{\infty} \frac{1}{z}\left[\left(\frac{1}{1+\Gamma \frac{1-\rho_{m}}{L G} z}\right)^{L}-\left(\frac{1}{1+\frac{1}{L}\left(\rho_{m}+\Gamma \frac{1-\rho_{m}}{G}\right) z}\right)^{L}\right] \\
& \times \prod_{k=1}^{K}\left(\frac{1}{1+\frac{1}{L G}\left[\frac{d_{k, m}}{d_{0, m}}\right]^{-\beta} z}\right)^{L} e^{-z \frac{\rho m}{\mathrm{SNR}_{m}}} d z \\
& \lim _{z \rightarrow 0} \frac{\prod_{l=1}^{L} \frac{1}{1+\Gamma \frac{1-\rho_{m}}{G} \alpha_{l} z}-\prod_{l=1}^{L} \frac{1}{1+\left(\rho_{m}+\Gamma \frac{1-\rho_{m}}{G}\right) \alpha_{l} z}}{z}=\frac{d}{d z}\left[\prod_{l=1}^{L} \frac{1}{1+\Gamma \frac{1-\rho_{m}}{G} \alpha_{l} z}-\prod_{l=1}^{L} \frac{1}{1+\left(\rho_{m}+\Gamma \frac{1-\rho_{m}}{G}\right) \alpha_{l} z}\right]_{z=0} \\
& =-\sum_{l=1}^{L} \frac{M-1}{G}\left(1-\frac{1}{L}\right) \alpha_{l}+\sum_{l=1}^{L}\left(1+\frac{M-1}{G}\left(1-\frac{1}{L}\right)\right) \alpha_{l}=\sum_{l=1}^{L} \alpha_{l} \\
& C_{m}=\mathbb{E}\left[\log _{2}\left(1+\frac{\xi_{0} \rho_{m} \sum_{l=1}^{L}\left|h_{l, 0}\right|^{2}}{\Gamma \frac{1-\rho_{m}}{G} \xi_{0} \sum_{l=1}^{L}\left|h_{l, 0}\right|^{2}+\frac{1}{G} \sum_{k=1}^{K} \sum_{l=1}^{L} \xi_{k}\left[\frac{d_{k, m}}{d_{0, m}}\right]^{-\beta}\left|h_{l, k}\right|^{2}+\frac{\rho_{m}}{\mathrm{SNR}_{m}}}\right)\right] \\
& C_{m}\left(\xi_{0}, \xi_{1}, \ldots, \xi_{K}\right)=\left(\log _{2} e\right) \int_{0}^{\infty} \frac{1}{z}\left[\prod_{l=1}^{L} \frac{1}{1+\Gamma \frac{1-\rho_{m}}{G} \alpha_{l} z \xi_{0}}-\prod_{l=1}^{L} \frac{1}{1+\left(\rho_{m}+\Gamma \frac{1-\rho_{m}}{G}\right) \alpha_{l} z \xi_{0}}\right] \\
& \times \prod_{k=1}^{K} \prod_{l=1}^{L} \frac{1}{1+\frac{1}{G}\left[\frac{d_{k, m}}{d_{0, m}}\right]^{-\beta} \alpha_{l} z \xi_{k}} e^{-z \frac{\rho_{m}}{\mathrm{SNR}_{m}}} d z
\end{aligned}
$$

regular hexagonal cell structure. The coordinates of the $K$ base stations are shown in Fig. 1 (in a hexagonal coordinates system).

In our numerical examples, we consider a CDMA system with $G=64$, and focus on a multipath channel with path loss-exponent $\beta=4$, and having exponential delay profile, where

$$
\alpha_{l, k}=\alpha_{1} e^{-\delta l}, \quad l=1,2, \ldots, L
$$

and $\delta$ is the decay rate. For the purpose of numerical examples, we assume that the reference mobile are located at the vertex of the home cell (this is the worst case interference scenario). Furthermore, unless otherwise state, we assume a uniform power allocations, such that $\rho_{1}=\rho_{2}=\ldots=\rho_{M}=1 / M$, with $M=10$ being the number of mobiles per cell.

Firstly, we validate the new theoretical analysis and assess the accuracy of approximating the orthogonality factor (3) by its average (4). Monte Carlo simulation results are given in Tables I and II for the uniform and exponential power delay profiles, respectively. Two different simulation results are shown in Tables I and II. The true random OF (which is given in (3)) is used in the simulation-I results, whereas the simulation-II and the theoretical results are based on the average OF (as given (4)). The first observation that can be made from Tables I and II is the excellent match between the theoretical and the simulation-II results. This validates the accuracy of the theoretical analysis that leads
TABLE I

SPECTRAL EFFICIENCY IN THE CASE OF A UNIFORM POWER DELAY PROFILE

\begin{tabular}{c|c|c|c|c} 
& & Simulation II & Simulation II & Theory \\
SNR $[\mathrm{dB}]$ & $L$ & Exact $\Gamma$, Eq. (3) & Approx. $\Gamma$, Eq. (4) & Eq. (12) \\
\hline \hline 3 & 2 & 0.192195572 & 0.186827344 & 0.186761039 \\
3 & 5 & 0.223014923 & 0.218202783 & 0.218439037 \\
3 & 10 & 0.234250533 & 0.231237734 & 0.231159274 \\
15 & 2 & 0.256539697 & 0.246243031 & 0.246526605 \\
15 & 5 & 0.250596359 & 0.244283089 & 0.244502853 \\
15 & 10 & 0.248155759 & 0.244474655 & 0.244559247 \\
\hline \hline
\end{tabular}

to (12). Moreover, comparing the simulation-I results with the corresponding simulation-II results (or equivalently, the new theoretical results) reveals that the spectral efficiency results obtained by using the average OF can never exceed the corresponding results obtained by using the true random $\mathrm{OF}$.

Therefore, we conclude that approximating the OF by its average will result in a tight lower bound on the spectral efficiency. This observation can be analytically proven by observing that the function $\log _{2}\left(1+\frac{a}{a x+b}\right)$ is a convex function $^{2}$ for all non-negative $a, b, x$. Therefore, Jenzen inequality asserts that, when $a$ and $b$ are non-negative constants and $x$ is

\footnotetext{
${ }^{2}$ Notice that $\frac{d^{2}}{d x^{2}} \ln \left(1+\frac{a}{a x+b}\right)>0, \forall$ non-negative $a, x, b$
} 


$$
\begin{aligned}
C_{m}=\left(\log _{2} e\right) \int_{0}^{\infty} \frac{1}{z}\left\{\sum _ { n = 1 } ^ { N } \frac { w _ { n } } { \sqrt { \pi } } \left[\prod_{l=1}^{L} \frac{1}{1+\Gamma \frac{1-\rho_{m}}{G} 10^{\left(\sqrt{2} \sigma a_{n}+\mu\right) / 10} \alpha_{l} z}\right.\right. \\
\left.\left.-\prod_{l=1}^{L} \frac{1}{1+\left(\rho_{m}+\Gamma \frac{1-\rho_{m}}{G}\right) 10^{\left(\sqrt{2} \sigma a_{n}+\mu\right) / 10} \alpha_{l} z}\right]\right\} \\
\quad \times \prod_{k=1}^{K}\left\{\sum_{n=1}^{N} \frac{w_{n}}{\sqrt{\pi}} \prod_{l=1}^{L} \frac{1}{1+\frac{1}{G}\left[\frac{d_{k, m}}{d_{0, m}}\right]^{-\beta} 10^{\left(\sqrt{2} \sigma a_{n}+\mu\right) / 10} \alpha_{l} z}\right\} e^{-z \frac{\rho_{m}}{\mathrm{SNR}_{m}} d z}
\end{aligned}
$$

TABLE II

\begin{tabular}{|c|c|c|c|c|}
\hline SNR [dB] & $L$ & $\begin{array}{r}\text { SimULATION I } \\
\text { Exact } \Gamma, \text { Eq. (3) } \\
\end{array}$ & $\begin{array}{c}\text { SIMULATION II } \\
\text { Approx. } \Gamma, \text { Eq. (4) }\end{array}$ & $\begin{array}{l}\text { THEORY } \\
\text { Eq. (12) } \\
\end{array}$ \\
\hline 3 & $\overline{2}$ & 0.171659106 & 0.168562899 & 0.168487128 \\
\hline 3 & 5 & 0.17989781 & 0.176049343 & 0.17607526 \\
\hline 3 & 10 & 0.179908998 & 0.176552925 & 0.176440111 \\
\hline 15 & 2 & 0.254897272 & 0.247625158 & 0.247676575 \\
\hline 15 & 5 & 0.252313543 & 0.245426161 & 0.245752504 \\
\hline 15 & 10 & 0.252125771 & 0.245668185 & 0.245691463 \\
\hline
\end{tabular}

SPECTRAL EFFICIENCY IN THE CASE OF AN EXPONENTIAL POWER DELAY PROFILE WITH DECAY RATE $\delta=1$.

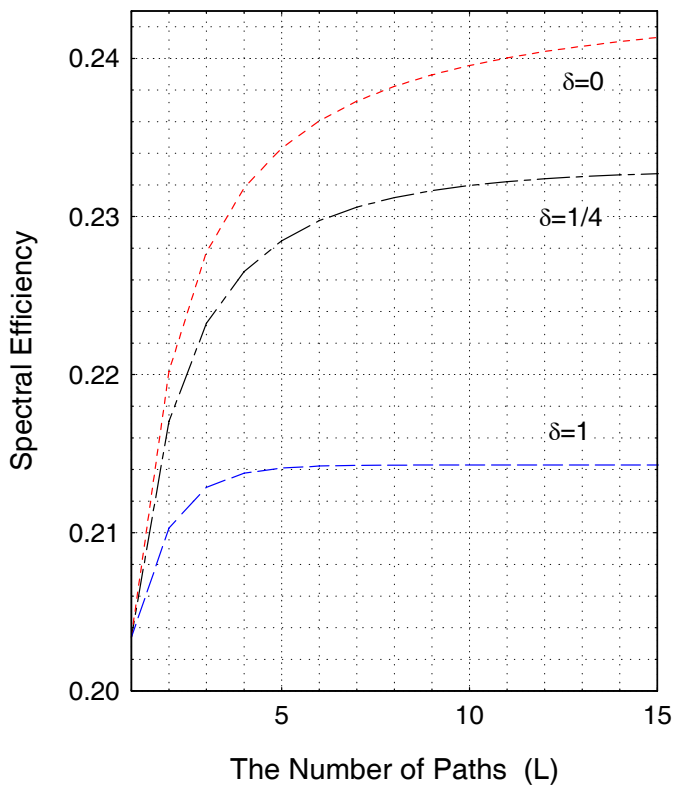

Fig. 2. Spectral efficiency $[\mathrm{b} / \mathrm{s} / \mathrm{Hz}]$ against the number of paths $(L)$ when $\mathrm{SNR}=7[\mathrm{~dB}]$ for several decay rates $\delta \in\{0,1 / 4 / 1\}$.

a non-negative random variable,

$$
\mathbb{E}\left[\ln \left(1+\frac{a}{a x+b}\right)\right] \geq \ln \left(1+\frac{a}{a \mathbb{E}[x]+b}\right) .
$$

This implies that, as far as the spectral efficiency is concerned, the approximation of the OF by its average will always result in a tight lower bound on the spectral efficiency.

In Figs. 2-5, we focus on the effects of multipath fading on the overall spectral efficiency of the downlink CDMA systems. In Figs. 2 and 3, we plot the spectral efficiency against the number of paths $L$ for two different values of SNR; $7 \mathrm{~dB}$ and $15 \mathrm{~dB}$, respectively. We observe from Figs. 2 and 3 that the spectral efficiency of the downlink CDMA system can

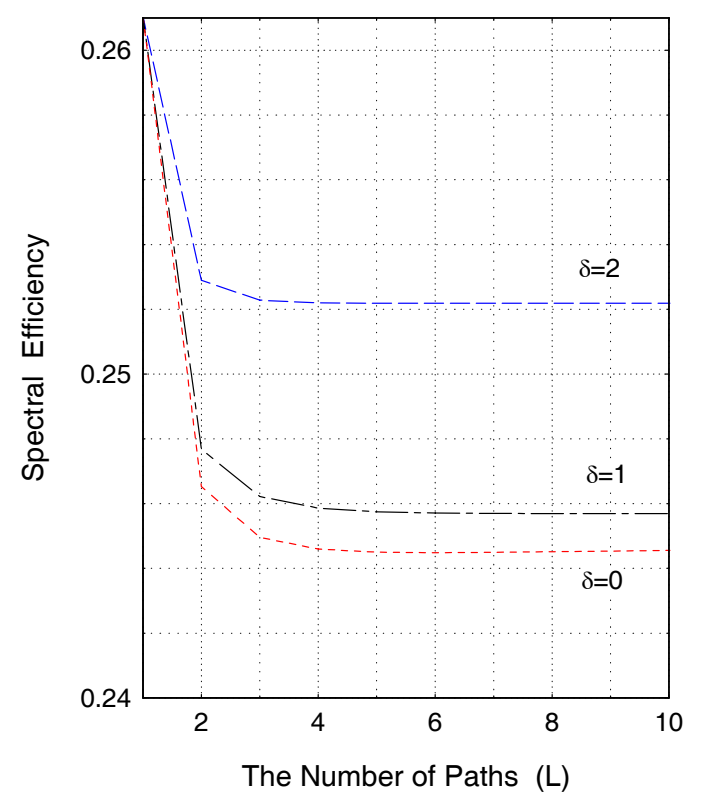

Fig. 3. The spectral efficiency $[\mathrm{b} / \mathrm{s} / \mathrm{Hz}]$ against the number of paths $(L)$ when $\mathrm{SNR}=15[\mathrm{~dB}]$, and for several decay rates $\delta \in\{0,1,2\}$.

be affected by varying the number of paths of the multipath fading channel in a two contradicting ways that depend on the SNR levels as follows. At low SNR, the spectral efficiency is increased with increasing the number of paths, whereas the opposite behavior is observed at high SNR levels. This observation is further demonstrated in Figs. 4 and 5, where the spectral efficiency is plotted against the SNR for the exponential and uniform power delay profiles, respectively.

In order to explain these observations, consider the expression of SINR, (1), and notice that increasing the number of paths can have two contradicting effects as follows; On one hand, it will increase the diversity order (more paths will contribute to the useful signal at the numerator of (1)). On the other hand, a non-zero self interference (the first term in the denominator of SINR in (1)) will appear when $L>1$ (where the amount of self interference will increase with increasing $L$, as can be observed from (3) or (4)). Therefore, the net effect will depend on which of these two factors outweigh the other. Now, looking at the first and last terms in the denominator of SINR in (1), one can see that the first term (the self-interference) becomes insignificant at low SNR levels (when compared to the last term, which is the reciprocal of the SNR), and therefore the diversity order dominates the loss 


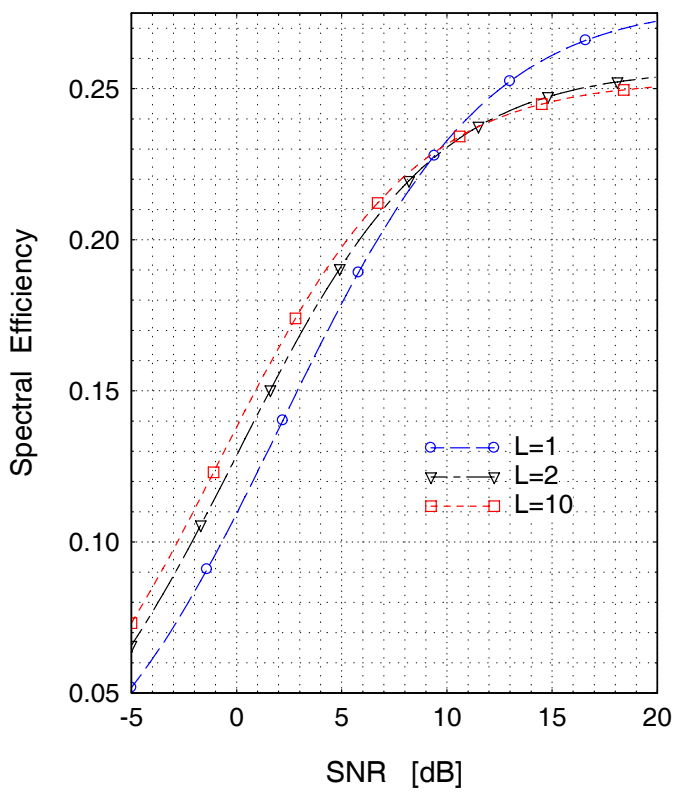

Fig. 4. The spectral efficiency $[\mathrm{b} / \mathrm{s} / \mathrm{Hz}]$ against $\mathrm{SNR}$ in $[\mathrm{dB}]$ for several number of paths $L \in\{1,2,10\}$, and for decay rate $\delta=1$.

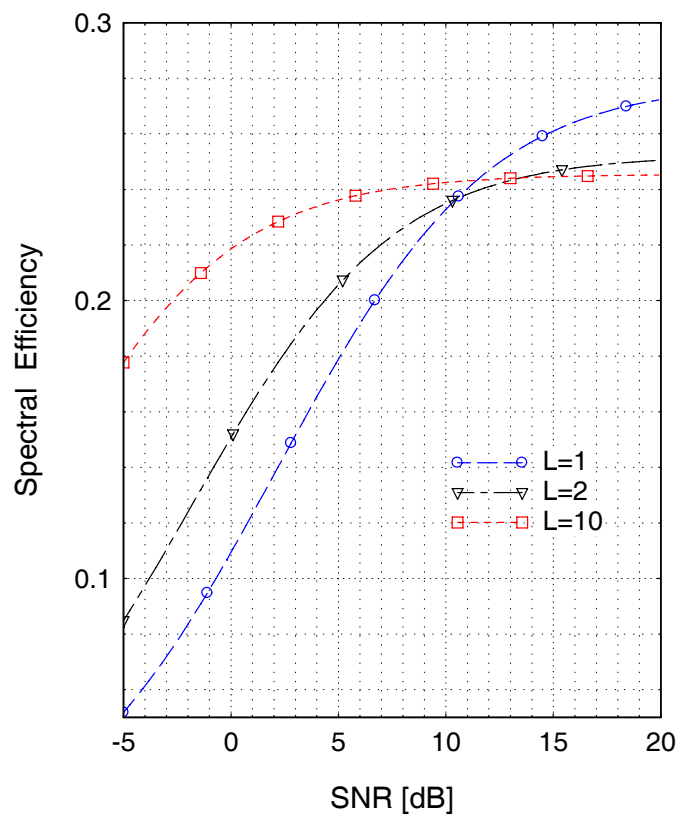

Fig. 5. The spectral efficiency in $[\mathrm{b} / \mathrm{s} / \mathrm{Hz}]$ against $\mathrm{SNR}$ in $[\mathrm{dB}]$ for several number of paths $L \in\{1,2,10\}$, and uniform power delay profile $(\delta=0)$.

of orthogonality at low SNR levels. On the other hand at high values of SNR, the last term becomes insignificant when compared to the self-interference term, and therefore the selfinterference will be dominant at high SNR levels.

In Fig. 6, we consider the effect of different power allocation strategies on the overall spectral efficiency. Here, the number of users is $M=20$, and we assume that the $m$ th mobile is located at $\left(\frac{m}{3 M}, \frac{m}{3 M}\right), m=1,2, \ldots, M$. In this case the distance between the $m$ th mobile and the home base station is $d_{0, m}=\frac{m}{M}$. Two power allocation strategies are shown in Fig. 6; $A$ is the perfect power control strategy, where

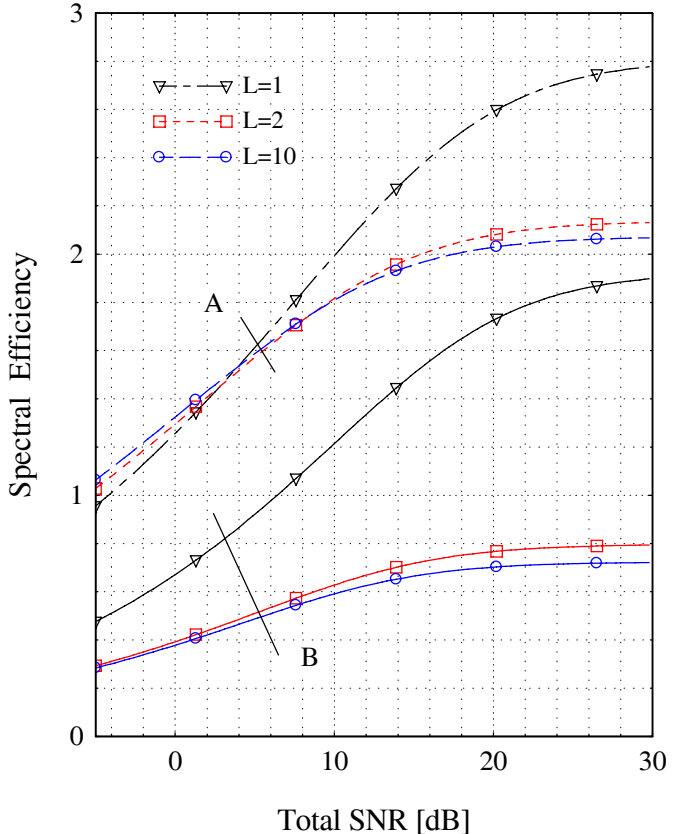

Fig. 6. Spectral efficiency against the total SNR, SNR $=\frac{P}{N_{0}}$ for two different power allocation strategies. $A$ is perfect power control strategy, and $B$ : is the uniform power allocation strategy. Exponential power delay profile with decay factor $\delta=1$.

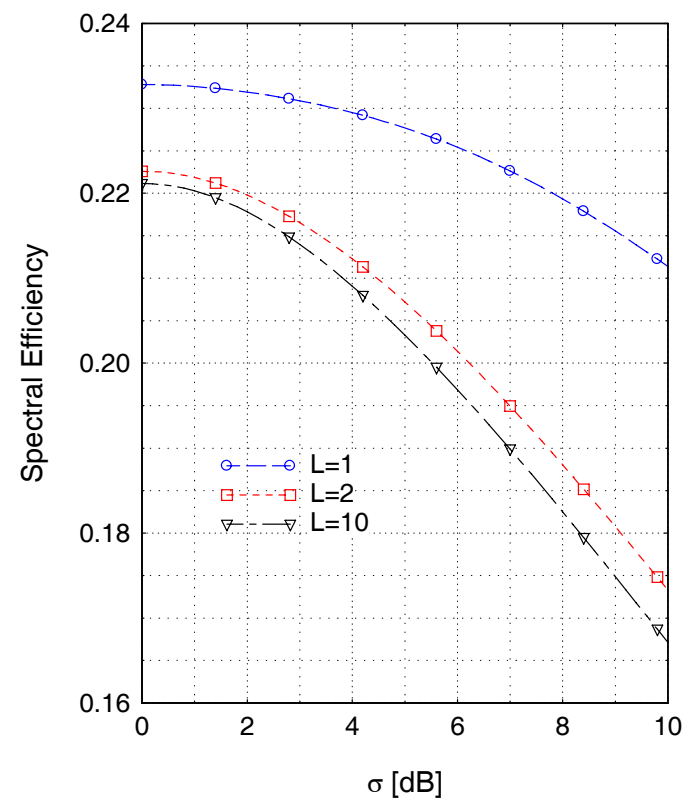

Fig. 7. Spectral efficiency against the standard deviation $[\mathrm{dB}\}$ of the lognormal shadow fading. $\mathrm{SNR}=10 \mathrm{~dB}$, and $\delta=1$.

$\rho_{m}=\frac{d_{0, m}^{\beta}}{\sum_{i=1}^{M} d_{0, i}^{\beta}}, m=1,2, \ldots, M$. On the other hand, $B$ is the uniform power allocation strategy, where $\rho_{m}=\frac{1}{M}$, $m=1,2, \ldots, M$. A significant improvement on the overall spectral efficiency of downlink CDMA in the multipath fading is observed when strategy $A$ is used in stead of the uniform allocation strategy $B$ that can exceed $200 \%$. For instance, at $\mathrm{SNR}=20 \mathrm{~dB}$, and $L=10$, Fig. 6 shows that the spectral efficiency increases from 0.7 in strategy $B$ into 2.0 in strategy $A$. 
Fig. 7 shows the performance of CDMA in the presence of log-normal shadow fading. Here, we plot the spectral efficiency against the standard deviation of the log-normal fading. We observe that multipath fading in the presence of shadow fading will deteriorate the spectral efficiency even more worst. For instance, as can be seen from Fig. 7, the spectral efficiency decreases in the absence of shadowing form 0.233 when $L=1$, into 0.221 when $L=10$ (which is equivalent to about $5 \%$ loss in the overall spectral efficiency. On the other hand, the spectral efficiency in the presence of shadow fading with standard deviation $\sigma=6 \mathrm{~dB}$ drops from 0.225 when $L=1$ to 0.197 when $L=10$ (which is equivalent to a drop that exceeds $12 \%$ in the overall spectral efficiency).

\section{Summary AND CONClusions}

In this paper, we have presented a new accurate theoretical analysis for the spectral efficiency of downlink multi-cell CDMA in the presence of multipath Rayleigh fading and lognormal shadowing. Based on an accurate interference model, we derive a new explicit expression for the spectral efficiency that accounts for both the interference from adjacent cells and the self-interference which would result form the loss of orthogonality. The numerical results has shown that the loss of orthogonality will deteriorate the spectral efficiency significantly at high SNR levels. On the other hand, the diversity gain of the multipath fading channel can be achieved at low SNR levels.

\section{APPENDIX A}

Let us invoke, firstly, the following identity

$$
\left(1+\frac{d u}{c u+v}\right)\left(1+\frac{c u}{v}\right)=1+\frac{(d+c) u}{v}
$$

which holds for any non-negative $c, u, v$, and implies that

$$
\ln \left(1+\frac{d u}{c u+v}\right)=\ln \left(1+\frac{(d+c) u}{v}\right)-\ln \left(1+\frac{c u}{v}\right) .
$$

Now, consider the integral

$$
\begin{aligned}
& \int_{0}^{\infty} \ln \left(1+\frac{c u}{v}\right) f(u ; a, m) d u \\
& =\int_{0}^{\infty} \ln \left(1+\frac{c u}{v}\right) \frac{u^{m-1}}{\Gamma(m)} a^{-m} e^{-u / a} d u
\end{aligned}
$$

which is reduced (by the change of variables $z=\frac{c u}{v}$ ) into

$$
\begin{aligned}
& \int_{0}^{\infty} \ln \left(1+\frac{c u}{v}\right) f(u ; a, m) d u \\
& =\int_{0}^{\infty} \ln (1+z) \frac{z^{m-1}}{\Gamma(m)}\left(\frac{v}{c a}\right)^{m} e^{-z \frac{v}{c a}} d z .
\end{aligned}
$$

Apply the rules of the integration by parts $m$ times to the integral in (29), to get

$$
\begin{aligned}
& \int_{0}^{\infty} \ln \left(1+\frac{c u}{v}\right) f(u ; a, m) d u \\
& =\int_{0}^{\infty}\left\{\frac{1}{\Gamma(m)} \frac{d^{m}}{d z^{m}} z^{m-1} \ln (1+z)\right\} e^{-z \frac{v}{c a}} d z
\end{aligned}
$$

Recall [18, Eq. 15.1.3] that

$$
\ln (1+z)=z_{2} F_{1}(1,1 ; 2 ;-z)
$$

where ${ }_{2} F_{1}(., . ; . ;$.$) is the Gauss hypergeometric function, and$ then apply [18, Eq. 15.2.3], to get [17]

$$
\begin{aligned}
\frac{1}{\Gamma(m)} & \frac{d^{m}}{d z^{m}} z^{m-1} \ln (1+z) \\
& =\frac{1}{\Gamma(m)} \frac{d^{m}}{d z^{m}} z^{m}{ }_{2} F_{1}(1,1 ; 2 ;-z) \\
& =m{ }_{2} F_{1}(1+m, 1 ; 2 ;-z) \\
& =m \int_{0}^{1}(1+t z)^{-(m+1)} d t \\
& =\frac{1}{z}-\frac{1}{z(1+z)^{m}}
\end{aligned}
$$

Recall [18, Eq. 15.3.1] was used in the third line of (32).

Therefore, we have from (30) and (32)

$$
\begin{aligned}
& \int_{0}^{\infty} \ln \left(1+\frac{c u}{v}\right) f(u ; a, m) d u \\
& =\int_{0}^{\infty} \frac{1}{z}\left(1-\frac{1}{(1+z)^{m}}\right) e^{-z \frac{v}{a c}} d z
\end{aligned}
$$

which is

$$
\begin{aligned}
& \int_{0}^{\infty} \ln \left(1+\frac{u}{v}\right) f(u ; a, m) d u \\
& =\int_{0}^{\infty} \frac{1}{z}\left(1-\frac{1}{(1+a c z)^{m}}\right) e^{-v z} d z
\end{aligned}
$$

Eq. (7) results straightforwardly from (34) and (27).

\section{REFERENCES}

[1] P. Varzakas and G. S. Tombras, "Spectral efficiency for a hybrid DS/FH code-division multiple-access system in cellular mobile radio," IEEE Trans. Veh. Technol., vol. 50, no. 6, pp. 1321-1327, Nov. 2001.

[2] M-S. Kwok, and H-S. Wang, "Adjacent cell interference analysis of reverse-link in CDMA cellular radio systems,"in IEEE Proc. PIMRC, vol. 2, Sept. 1995, pp. 446-450.

[3] J. Cho and D. Hong, "Downlink throughput and fairness analysis in multi-rate CDMA systems,"in Proc. IEEE VTC, vol. 2, May 2001, pp. 1001-1006.

[4] J. S Wu, J. K. Chung, and M. T. Sze, "Analysis of uplink and downlink capacities for two-tier cellular system,"IEE Proc. Commun., vol. 144, no. 6, pp. 405-412, Dec. 1997.

[5] D. L. Noneaker, "On the spectral efficiency of wideband CDMA systems,"IEEE J. Select. Areas Commun., vol. 19, no. 1, pp. 33-47, Jan. 2001.

[6] N. Bonneau, M. Debbah, and E. Altman, "Spectral efficiency of CDMA downlink cellular networks with matched filter,"EURASIP J. Wireless Commun. and Networking, vol. 2006, pp. 1-10, 2006.

[7] O. Ertug, B. S. Unal, and B. Baykal, "Spectral efficiency and errorexponents of randomly-spread CDMA with linear multiuser receivers over multipath Rayleigh fading channels," in Proc. Seminar on Broadband Commun., vol. 2, Feb. 2002, pp. 936-941.

[8] J. G. Proakis, Digital communications, 2nd edition. McGraw-Hill, 1989.

[9] P. M. Shankar, Introduction to Wireless Systems. John Wiley \& Sons, 2001.

[10] O. Awoniyi, N. B. Mehta, and L. J. Greenstein, "Characterizing the orthogonality factor in WCDMA downlinks,"IEEE Trans. Wireless Commun., vol. 2, no. 4, pp. 621-625, July 2003.

[11] N. B. Mehta, A. F. Molisch, and L. J. Greenstein, "Orthogonality factor in WCDMA downlinks in urban macrocellular environments,"in Proc. IEEE GLOBECOM., vol. 6, Nov. 2005, pp. 621-625.

[12] M. Hunukumbure, M. Beach, and B. Allen, "Downlink orthogonality factor in UTRA FDD systems,"IEEE Electron. Lett., vol. 38, no. 4, pp. 196-197, Feb. 2002.

[13] N. B. Mehta, L. J. Greenstein, T. M. Willis, and Z. Kostic, "Analysis and results for the orthogonality factor in WCDMA downlinks,"IEEE Trans. Wireless Commun., vol. 2, no. 6, pp. 1138-1149, Nov. 2003.

[14] S. Verdu and S. Shamai, "Spectral efficiency of CDMA with random spreading,"IEEE Trans. Inform. Theory, vol. 45, no. 2, pp. 622-640, Mar. 1999. 
[15] P. G. Moschopoulos, "The distribution of the sum of independent gamma random variables," Ann. Inst. Statist. Math. (Part A), vol. 37, pp. 541544, 1985.

[16] M.-S. Alouini, A. Abdi, and M. Kaveh, "Sum of gamma variates and performance of wireless communication systems over Nakagami-fading channels," IEEE Trans. Veh. Technol., vol. 50, no. 6, pp. 1471-1480, Nov. 2001.

[17] K.A. Hamdi, "A useful technique for interference analysis in Nakagami fading," IEEE Trans. Commun., vol. 55, no. 6, June 2007.
[18] S. M. Abramowitz, Handbook of Mathematical Functions with Formulas, Graphs, and Mathematical Tables. U.S. Department of Commerce, 1972.

[19] T. Eng and L. B. Milstein, "Coherent DS-CDMA performance in Nakagami multipath fading," IEEE Trans. Commun., vol. 43, no. 4, Apr. 1995.

[20] M. K. Simon and M.-S. Alouini, Digital Communication over Fading Channels, 2nd ed. Hoboken, NJ: Wiley-Interscience, 2005. 